\title{
Tracheostomy after pediatric cardiac surgery: Frequency, indications, and outcomes
}

\author{
Timothy Cotts, MD, ${ }^{\mathrm{a}}$ Jennifer Hirsch, MD, MS, ${ }^{\mathrm{b}}$ Marc Thorne, MD, MPH, ${ }^{\mathrm{c}}$ and Robert Gajarski, $\mathrm{MD}^{\mathrm{a}}$
}

Objectives: This study was designed to review baseline characteristics and outcomes of children requiring tracheostomy after cardiac surgery.

Methods: A retrospective review of children under age 2 requiring tracheostomy after cardiac surgery between January 1999 and December 2005 was performed. Indications for tracheostomy, survival, and completion of staged palliation were documented.

Results: After cardiac surgery, $59(1.3 \%)$ of 4503 patients with a median age at surgery of 15 days and weight of $3.5 \mathrm{~kg}$ required tracheostomy. Median duration from surgery to tracheostomy was 36 days (range 10-145 days). Genetic syndromes or major noncardiac comorbidities were present in $40 \%$ of patients. Biventricular repair was performed in 34 patients and univentricular repair in 25. Tetralogy of Fallot variants $(29 \%)$ and coarctation \pm ventricular septal defect $(21 \%)$ constituted the majority of biventricular lesions associated with tracheostomy, whereas unbalanced atrioventricular septal defect and hypoplastic left heart syndrome with highly restrictive atrial septal defect accounted for $52 \%$ of the single ventricle group. Indications for tracheostomy included the following: multifactorial (37\%), tracheobronchomalacia, $(24 \%)$, cardiac (12\%), bilateral vocal cord paralysis $(10 \%)$, bilateral diaphragm paralysis $(2 \%)$, and other airway issues (15\%). Hospital survival was $75 \%$ with intermediate-term (median, 25.5 months; range, $1-122$ months) survival of $53 \%$. Of 25 single ventricle patients, $6(24 \%)$ had successful completion of the Fontan procedure. Of 12 patients with single ventricle who were ventilator-dependent after initial repair, 10 died, 1 remains at hemi-Fontan, and 1 has undergone completion of the Fontan procedure.

Conclusions: Requirement for tracheostomy in pediatric patients after cardiac surgery was associated with significant mortality. Patients with single ventricle have the highest late death rate and those with chronic ventilator dependency were unlikely to undergo successful Fontan completion. (J Thorac Cardiovasc Surg 2011;141:413-8)

Earn CME credits at

http://cme.ctsnetjournals.org

Although the vast majority of children undergoing cardiovascular surgery tolerate early extubation, some require prolonged ventilatory support and are unable to be weaned from mechanical ventilation. Such patients often require tracheostomy to facilitate long-term mechanical ventilation. Other patients may have congenital airway anomalies, or postoperative airway complications may develop, which necessitate tracheostomy for airway maintenance. Additionally, many children undergoing surgery for congenital heart disease have genetic syndromes such as 22q11 deletion syndrome, which can be associated with airway and facial abnormalities. Previous

\footnotetext{
From the Division of Cardiology, ${ }^{a}$ Department of Pediatrics, the Section of Cardiac Surgery, ${ }^{b}$ Department of Surgery, and the Division of Pediatric Otolaryngology, ${ }^{\mathrm{c}}$ Department of Otolaryngology, University of Michigan Medical School, Ann Arbor, Mich. Disclosures: Authors have nothing to disclose with regard to commercial support

Received for publication Feb 18, 2010; revisions received April 30, 2010; accepted for publication June 20, 2010; available ahead of print July 20, 2010.

Address for reprints: Timothy Cotts, MD, L1242 Women's Box 5204, 1500 E Medical

Center Dr, Ann Arbor, MI 48109-5204 (E-mail: cottstim@umich.edu). $0022-5223 / \$ 36.00$

Copyright (c) 2011 by The American Association for Thoracic Surgery doi:10.1016/j.jtcvs.2010.06.027
}

studies have shown that airway issues prolong mechanical ventilation and increase length of stay in the intensive care unit (ICU) in infants after cardiac surgery. ${ }^{1-3}$ Additionally, infants and children undergoing cardiac surgery, particularly patients with single ventricle undergoing multiple repairs, are at risk for surgical complications that increase the risk for respiratory compromise. These include hemidiaphragm paralysis and vocal cord paralysis for those patients undergoing complex aortic arch repairs.

Early reports did not demonstrate increased mortality with prolonged mechanical ventilation, although more recent reports have demonstrated increased mortality and prolonged ICU stay in neonates undergoing cardiac surgery. ${ }^{4,5}$ Early tracheostomy is common in adults after cardiac surgery, with data demonstrating higher mortality and ICU length of stay in patients undergoing delayed tracheostomy. ${ }^{6}$ There are no established criteria or routine practices in the pediatric population. Data from the pediatric population are limited to small, single-center reviews, with results highly dependent on the patient population and practice patterns of the particular study center. ${ }^{7-9}$ There are no studies specifically examining outcomes of patients with single ventricle, including Fontan completion rates. Inasmuch as tracheostomy is likely a surrogate for poor hemodynamic or pulmonary status, it is likely to predict patient outcomes including the ability to proceed successfully through the Fontan procedure. The 


\section{Abbreviations and Acronyms \\ HLHS = hypoplastic left heart syndrome \\ ICU = intensive care unit \\ VACTERL $=$ vertebral abnormalities, anal atresia, cardiac abnormalities, tracheoesophageal fistula and/or esophageal atresia, renal agenesis and dysplasia, and limb defects}

aims of this study were to describe the indications for tracheostomy in infants and children undergoing cardiac surgery, as well as the outcomes of these patients, particularly those with single ventricle physiology.

\section{METHODS}

A retrospective review was performed at a single center. Patient admissions to the congenital heart center service were identified using ICD-9 tracheostomy codes. All children less than 2 years old with congenital heart disease undergoing tracheostomy between January 1999 and December 2005 were studied. The vast majority of patients underwent diagnostic bronchoscopic examination before or at the time of tracheostomy. Institutional Review Board approval from the University of Michigan was obtained with waiver of informed consent.

\section{Baseline Data}

Baseline data including demographics, cardiac diagnosis, and surgical procedure performed were documented. If multiple cardiac anomalies were present, the most hemodynamically significant lesion was used. Presence of genetic syndromes and other noncardiac comorbidities were also recorded. Comorbidities were included if they required intervention in the neonatal period or infancy (ie, tracheoesophageal fistula or congenital diaphragmatic hernia).

\section{Indications for Tracheostomy}

Potential indications for tracheostomy included tracheobronchomalacia, tracheal and subglottic stenosis, vocal cord paralysis, other upper airway issues, suboptimal hemodynamics, and diaphragm paralysis. The indication for tracheostomy was determined by a panel of reviewers consisting of 2 pediatric cardiac intensivists (T.C. and R.G.) and an attending pediatric otolaryngologist (M.T.). For patients in whom one precise etiology could not be determined or agreed on, the indication was deemed multifactorial and the likely factors were explicitly stated. Suboptimal hemodynamic status was defined as moderate or worse systemic ventricular function, moderate or greater atrioventricular valve insufficiency, significant hypoxemia, or other significant residual disease. These indications were labeled "cardiac" for the purposes of this analysis.

\section{Outcome Measures}

Short-term survival (ie, survival to hospital discharge) was recorded. Intermediate-term survival was defined as survival to most-recent followup. The need for mechanical ventilation at the time of hospital discharge was also documented. The records for patients undergoing staged palliation were examined to determine whether patients successfully completed their palliative series, including successful completion of the Fontan procedure. Finally, records were reviewed to determine whether patients had been successfully decannulated and, if so, to document the time from the date of tracheostomy to decannulation.

\section{Statistical Analysis}

Statistical analysis was performed using GraphPad InStat version 3.06 (GraphPad Software, San Diego, Calif). Medians (ranges) or means (standard deviations) were used to express descriptive data. Categorical data were assessed using contingency tables and $\chi^{2}$ or Fisher's exact test as appropriate.

\section{RESULTS}

\section{Baseline Characteristics}

A total of 4503 cardiac procedures were performed in children less than 2 years of age during the 7-year study period. A total of $59(1.3 \%)$ patients required tracheostomy after cardiac surgery. Of these, 25 (11 girls) patients had single ventricle anatomy, and 34 (16 girls) had biventricular anatomy. The median age at surgery was 15 days (range, 1-581 days), with a median weight of $3.5 \mathrm{~kg}$ (range, $1.9-11.1 \mathrm{~kg}$ ). The median duration from the date of surgery to tracheostomy was 36 days (range, $10-145$ days) and the median length of hospital stay was 62 days (range, 6-345 days). Median duration of follow-up for survivors to hospital discharge was 47 months (range, 1-122 months). One patient was lost to follow-up after transfer to an outside hospital. A total of $24(40 \%)$ patients had a genetic syndrome or major noncardiac comorbidity. Common genetic syndromes included Down syndrome in 5 patients, DiGeorge syndrome in 4 patients, and VACTERL (vertebral abnormalities, anal atresia, cardiac abnormalities, tracheoesophageal fistula and/or esophageal atresia, renal agenesis and dysplasia, and limb defects) in 4 patients. Other chromosomal abnormalities of unclear significance (unbalanced translocation of chromosomes 4 and 8 , as well as a partial duplication of chromosome 9) were also seen. In terms of noncardiac issues, Pierre Robin sequence was present in 4 patients, omphalocele in 2 patients, and Dandy-Walker malformation, large encephalocele, and congenital rubella syndrome in 1 patient each. Of the 25 patients with single ventricle, $4(16 \%)$ had a genetic syndrome or major comorbidity compared with 20 of 34 (59\%) patients undergoing biventricular repairs $(P=.0012)$.

The underlying cardiac diagnoses for patients requiring tracheostomy are shown in Table 1. A large proportion of patients $(35 \%)$ had conoventricular lesions including tetralogy of Fallot with or without associated lesions including absent pulmonary valve, pulmonary atresia, and interrupted aortic arch. Coarctation of the aorta with or without ventricular septal defect accounted for $21 \%$ of patients, and vascular rings and slings accounted for an additional $12 \%$ of patients requiring tracheostomy. High-risk single ventricle anatomy comprised a disproportionate degree of patients with single ventricle who required tracheostomy. Of 25 patients with single ventricle, 7 had complete atrioventricular septal defect, 6 hypoplastic left heart syndrome (HLHS) with highly restrictive or intact atrial septa, 1 HLHS with moderate-severe tricuspid regurgitation, 1 with tricuspid 
TABLE 1. Underlying diagnosis for patient undergoing tracheostomy $(\mathbf{n}=\mathbf{5 9})$

\begin{tabular}{|c|c|}
\hline Biventricular repairs & $\mathbf{n}=\mathbf{3 4}$ \\
\hline Conotruncal defects & 12 \\
\hline Tetralogy of Fallot & 4 \\
\hline Tetralogy of Fallot/absent pulmonary valve & 3 \\
\hline Tetralogy of Fallot/pulmonary atresia & 3 \\
\hline Common arterial trunk & 1 \\
\hline Common arterial trunk/interrupted aortic arch & 1 \\
\hline Interrupted aortic arch & 1 \\
\hline Coarctation of the aorta & 7 \\
\hline Coarctation of the aorta/VSD & 4 \\
\hline Coarctation of the aorta & 3 \\
\hline Lesions with extracardiac vascular compression & 4 \\
\hline Pulmonary artery sling/VSD/ASD & 1 \\
\hline Pulmonary artery sling/heterotaxy/VSD & 1 \\
\hline Double aortic arch with AVSD & 1 \\
\hline Double aortic arch & 1 \\
\hline VSD & 3 \\
\hline TAPVC & 2 \\
\hline AVSD & 1 \\
\hline AVSD/TAPVC & 1 \\
\hline CCTGA, pulmonary atresia & 1 \\
\hline HLHS s/p heart transplant & 1 \\
\hline D-TGA & 1 \\
\hline Single ventricle palliations & $\mathbf{n}=\mathbf{2 5}$ \\
\hline HLHS & 7 \\
\hline Unbalanced AVSD & 7 \\
\hline HLHS with highly restrictive or intact atrial septum & 6 \\
\hline Other complex single ventricle & 3 \\
\hline HLHS with $>$ moderate tricuspid insufficiency & 1 \\
\hline Tricuspid atresia & 1 \\
\hline
\end{tabular}

atresia, 7 with "straightforward" HLHS anatomy, and 3 with other complex single ventricle anatomy. During the study period, a total of 358 Norwood procedures were performed for all variants of HLHS, including those with unbalanced atrioventricular septal defect. The rate of tracheostomy for this group was 3.6\% (13/358).

\section{Indications for Tracheostomy}

The indications for tracheostomy in patients with biventricular and single ventricle anatomies are listed in Table 2. For the biventricular repair group $(\mathrm{n}=34)$, tracheobronchomalacia (including that caused by extrinsic vascular compression) was the primary factor in 14 patients. Multiple factors were present in 9 patients, whereas primary cardiac causes and other airway issues were factors in a small proportion of patients.

A multifactorial etiology was present in over half of the patients with single ventricle, with most of the multifactorial indications including suboptimal hemodynamics in conjunc-
TABLE 2. Indications for tracheostomy for patients undergoing biventricular repairs $(n=34)$

\begin{tabular}{lr}
\hline \multicolumn{1}{c}{ Postoperative factor } & N (\%) \\
\hline TBM/vascular compression & $14(41)$ \\
Multifactorial & $9(26)$ \\
TBM + VCP (3) & \\
Cardiac + DP (3) & \\
Cardiac + subglottic stenosis (1) & \\
Cardiac + TBM (1) & \\
DP + TBM (1) & $4(12)$ \\
Tracheal/subglottic stenosis & $4(12)$ \\
Cardiac & $3(9)$ \\
Other upper airway & \\
\hline Postoperative factors contributing to requirement for tracheostomy in infants undergo- \\
ing biventricular repairs. $T B M$, Tracheobronchomalacia; $V C P$, vocal cord paralysis; \\
$D P$, diaphragm paralysis.
\end{tabular}

tion with an operative complication such as vocal cord (bilateral in 5/6 patients) or bilateral diaphragmatic paralysis (Table 3). Tracheobronchomalacia was not seen as a solitary indication for tracheostomy in patients undergoing single ventricle palliations.

\section{Detailed Results for Vascular Ring Population}

A total of 49 repairs of vascular rings and pulmonary artery slings were performed during the course of the study period. The anatomy consisted of pulmonary artery sling in 4 patients, double aortic arch in 18 patients, and right aortic arch with aberrant subclavian artery in 27 patients. An additional 2 patients had upper airway obstruction (Pierre Robin sequence) and underwent tracheostomy before vascular ring surgery; therefore, they were not included in the analysis. A total of 8 patients underwent intracardiac repairs at the time of their ring repair.

Among the 4 patients with vascular rings requiring tracheostomy, the diagnosis was pulmonary artery sling in 2 patients and double aortic arch in 2 patients. Both of the

TABLE 3. Postoperative factors for patients undergoing single ventricle palliations $(n=25)$

\begin{tabular}{lc}
\hline \multicolumn{1}{c}{ Postoperative factor } & $\mathbf{n}(\%)$ \\
\hline Multifactorial & $13(52)$ \\
Cardiac + DP (6) & \\
Cardiac + VCP (3) & \\
Cardiac + DP + VC (2) & \\
Cardiac + TBM + VC (1) & \\
TBM + DP (1) & $6(24)$ \\
Vocal cord paralysis* & $3(12)$ \\
Cardiac & $1(4)$ \\
Diaphragm paralysis (bilateral) & $1(4)$ \\
Subglottic stenosis & $1(4)$ \\
Glossoptosis (Pierre Robin) & \\
\hline Postoperative factors contributing to requirement for tracheostomy in infants undergo- \\
ing single ventricle palliations. $D P$, Diaphragm paralysis; $V C P$, vocal cord paralysis; \\
TBM, Tracheobronchomalacia. *Vocal cord paralysis was bilateral in 5 of 6 patients.
\end{tabular}


patients with a pulmonary artery sling had long-segment tracheal stenosis and required tracheal reconstruction at the time of repair. Both of these patients had major additional lesions, 1 with VACTERL syndrome, ventricular septal defect, and atrial septal defect, and the other with heterotaxy syndrome, ventricular septal defect, and absent left lung. The latter patient also underwent aortopexy at the time of repair. Of the 2 patients with double aortic arch requiring tracheostomy, 1 was an infant with Down syndrome, atrioventricular septal defect, and severe tracheal stenosis. The second patient with double aortic arch had a lifethreatening respiratory event after surgery and underwent trachesotomy for severe tracheomalacia.

\section{Complications of Tracheostomy}

A standardized approach to repeat sternotomy after tracheostomy was used. The tracheostomy site was draped outside the surgical field with a sterile impermeable drape. No changes were made in prophylactic antibiotic therapy. The incidence of wound infections in the single ventricle population was assessed, inasmuch as a large percentage of these patients underwent subsequent sternotomy with a trachesotomy in place (14 patients underwent 22 sternotomies with a tracheostomy in place). Three cases of mediastinitis were seen, 2 involving Pseudomonas aeruginosa and 1 involving Escherichia coli. Two of these patients were critically ill postoperatively and were left with the sternum open for prolonged periods ( $>7$ days).

\section{Mortality}

The overall survival to hospital discharge or transfer was $46(78 \%)$ of 59 , with $27(79 \%)$ of 34 surviving in the biventricular repair group, and $19(76 \%)$ of 25 surviving in the single ventricle group. Overall survival during the follow-up period was $31(53 \%)$ of 58 , with $22(67 \%)$ of 33 surviving in the biventricular repair group (1 patient lost to follow-up) and $9(36 \%)$ of 25 in the single ventricle group $(P=.03)$.

Among all patients with congenital heart disease undergoing tracheostomy, $23(40 \%)$ of 58 were decannulated after a median time of 32 months (range, 3-98 months). For patients with single ventricle, $9(36 \%)$ of 25 were successfully decannulated after 26 months (range, 5-59 months) compared with $14(42 \%)$ of 33 patients in the biventricular repair group after 33 months (range, $14-33$ months; $P=.62$ ).

\section{Detailed Results for Patients With Single Ventricle}

Patients undergoing single ventricle palliations requiring tracheostomy were divided into 2 groups. The first group (non-ventilator dependent) included patients with isolated upper airway causes for tracheostomy who did not require mechanical ventilation at discharge; the second group (ventilator dependent) could not be weaned from mechanical ventilation and were, therefore, ventilated at the time of

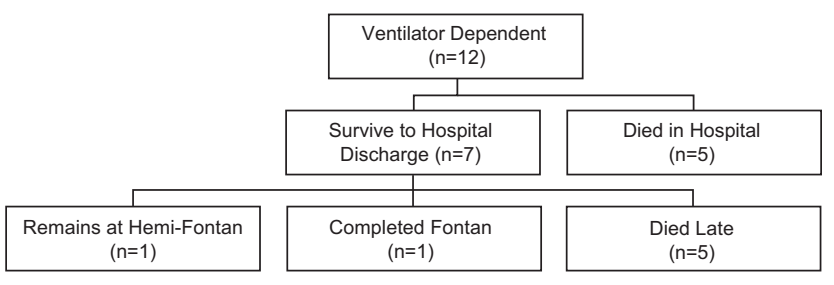

FIGURE 1. Outcomes of patients with single ventricle who were ventilator-dependent at time of hospital discharge.

death or discharge from the hospital. A flow diagram for this group is shown in Figure 1. Initial hospital survival for patients not requiring mechanical ventilation was 12 $(92 \%)$ of 13 , compared with 7 (58\%) of 12 for those requiring mechanical ventilation $(P=.07)$. Five of the 13 nonventilator-dependent patients compared with 1 of 12 ventilator-dependent patients were able to have successful completion of the Fontan procedure $(P=.16)$.

\section{DISCUSSION}

This single-center study identified a low rate of tracheostomy $(0.9 \%)$ among patients after cardiac surgery, which is comparable with previously reported data. ${ }^{7}$ In addition, tracheostomy was associated with a high rate of morbidity and mortality, particularly among infants. Although this may not be surprising, these data do provide insight into patient and perioperative factors contributing to tracheostomy requirement, as well as outcomes for this patient population. This study is the largest to date and the first to detail outcomes after tracheostomy for patients with single ventricle.

\section{Two-Ventricle Repair Population}

Tracheobronchomalacia was a common factor in the need for tracheostomy in the biventricular repair group. An earlier study demonstrated increased mortality and prolonged hospital stay in children with tracheobronchomalacia. ${ }^{10}$ Interestingly, this study demonstrated a higher risk of death for redo procedures and palliative procedures in this group, demonstrating that the comorbidity of tracheobronchomalacia can contribute to mortality in this complex patient population. The other dominant finding among patients having biventricular repair was the high rate $(59 \%)$ of genetic syndromes and noncardiac anomalies. Syndromes commonly associated with congenital heart disease, including DiGeorge syndrome and Down syndrome, may be associated with increased risk for airway abnormalities. ${ }^{11,12}$ Additionally, major noncardiac anomalies such as congenital diaphragmatic hernia or tracheoesophageal fistula may increase the risk for prolonged mechanical ventilation or the need for tracheostomy.

\section{Single Ventricle Repair Population}

Two broad categories of patients with single ventricle required tracheostomy. The first included patients with 
relatively straightforward HLHS anatomy who had the surgical complications of vocal cord or hemidiaphragm paralysis. The incidence of vocal cord paralysis in this subgroup has been cited at $9 \%{ }^{13}$ and is likely the result of extensive aortic arch reconstruction performed at the time of the Norwood procedure with resultant recurrent laryngeal nerve injury. In this study, patients with isolated vocal cord paralysis who required tracheostomy solely for airway maintenance generally were long-term survivors and tolerated Fontan completion. This sharply contrasts with the second group of patients with single ventricle, which consisted of those with high-risk anatomy, including unbalanced atrioventricular septal defect, significant tricuspid insufficiency, or highly restrictive or intact atrial septum. In these patients, a multifactorial etiology of tracheostomy requirement could be seen, with additive insults of vocal cord and diaphragm paralysis in the setting of suboptimal hemodynamics. These patients have high mortality, with poor rates of completion of the Fontan procedure. Diaphragm paralysis is a known complication of cardiac surgery, with rates reported from $1.6 \%$ to $5.4 \% .{ }^{14-16}$ The prevalence in patients after Fontan procedure has been reported to be as high as $24.6 \%$ and is associated with increased morbidity. ${ }^{17}$ Minimizing the operative complications of vocal cord and diaphragm paralysis is imperative, particularly in patients already at increased risk. The patients at higher risk for mortality (those with unbalanced atrioventricular septal defect and those with highly restrictive atrial septa) may also be at high risk for these complications owing to atypical anatomy related to heterotaxy, as well as the need for multiple reoperations.

Inasmuch as this study was performed at a single institution, institutional practices likely are a large factor in terms of both the number of patients undergoing tracheostomy and their outcomes. There is no institutional protocol for the timing of tracheostomy in patients with congenital heart disease. If a patient cannot be extubated, the general approach is to identify anatomic or physiologic causes that are potentially reversible. For example, if a paralyzed hemidiaphragm is found, the patient would undergo diaphragm plication before a second extubation attempt. On average, patients are given 3 extubation attempts before tracheostomy, unless a problem is identified that is unlikely to improve (ie, severe subglottic stenosis). The median duration from cardiac surgery to tracheostomy of 36 days (range, 10-145 days) is only slightly longer than the 30-day duration previously published ${ }^{7}$ These durations are significantly longer than the standard practice of tracheostomy within 2 to 3 weeks in adult patients. This is supported by data demonstrating longer ICU stay, higher ICU mortality, and higher rate of failure to wean from mechanical ventilation in adults undergoing tracheostomy after greater than 21 days of intubation. ${ }^{6}$ Results of very early tracheostomy (within 2-4 days) in adults have been mixed. ${ }^{18,19}$ Given different etiologies for prolonged ventilation in adults compared with children, it is difficult to extrapolate experience from adult studies to the pediatric population. Although it is possible that early tracheostomy may improve outcomes in young children, which patients may benefit from this practice is unknown at this time.

The extremely poor outcomes of patients with single ventricle who require tracheostomy is concerning and requires further study. These patients often represent the highest risk single ventricle group, including those with unbalanced atrioventricular septal defect and tricuspid insufficiency, as well as those with highly restrictive or intact atrial septa. Consideration for earlier referral for cardiac transplantation or alternative operative approaches, such as a hybrid procedure, should be considered for these high-risk patients. Data for high-risk patients with single ventricle undergoing hybrid procedures are limited to small single-center studies, demonstrating $43 \%$ to $50 \%$ mortality at intermediate-term follow-up. ${ }^{20,21}$ If high-risk patients with single ventricle require tracheostomy and long-term mechanical ventilation, parents should be counseled/informed about the grim prognosis for this patient population.

\section{Limitations}

Inasmuch as this is a single-center study, the results may not necessarily be applicable to all centers. The study institution likely has a referral bias for high-risk patients with single ventricle inasmuch as many such patients present from outside the geographic referral area. Additionally, a large proportion of the group having biventricular repair had genetic syndromes or significant noncardiac comorbidities, which may induce referral bias as well. Inasmuch as this was a retrospective review, caution must be exercised before prospective application of the results.

\section{CONCLUSIONS}

Requirement for tracheostomy in pediatric patients after cardiac surgery was associated with significant early and late mortality. Patients with single ventricle have the highest late death rate and those with chronic ventilator dependency were unlikely to undergo successful Fontan completion. Further study of the risk factors for tracheostomy and poor outcomes in patients undergoing single ventricle palliation is necessary.

\section{References}

1. Bandla HP, Hopkins RL, Beckerman RC, Gozal D. Pulmonary risk factors compromising postoperative recovery after surgical repair for congenital heart disease. Chest. 1999;116:740-7.

2. Brown KL, Ridout DA, Goldman AP, Hoskote A, Penny DJ. Risk factors for long intensive care unit stay after cardiopulmonary bypass in children. Crit Care Med. 2003;31:28-33.

3. Székely A, Sápi E, Király L, Szatmári A, Dinya E. Interoperative and postoperative risk factors for prolonged mechanical ventilation after pediatric cardiac surgery. Paediatr Anaesth. 2006;16:1166-75.

4. Kanter RK, Bove EL, Tobin JR, Zimmerman JJ. Prolonged mechanical ventilation of infants after open heart surgery. Critical Care Med. 1986;14:211-4. 
5. Shi S, Zhao Z, Liu X, Shu Q, Tan L, Lin R. Perioperative risk factors for prolonged mechanical ventilation following cardiac surgery in neonates and young infants. Chest. 2008;134:768-74.

6. Hsu CL, Chen KY, Chang CH, Jerng JS, Yu CJ, Yang PC. Timing of tracheostomy as a determinant of weaning success in critically ill patients: a retrospective study. Crit Care. 2005;9:R46-52.

7. Hoskote A, Cohen G, Goldman A, Shekerdemian L. Tracheostomy in infants and children after cardiothoracic surgery: indications, associated risk factors, and timing. J Thorac Cardiovasc Surg. 2005;130:1086-93.

8. LoTempio MM, Shapiro NL. Tracheostomy tube placement in children following cardiothoracic surgery: indications and outcomes. Am J Otolaryngol. 2002;23:337-40.

9. Rossi AF, Fishberger S, Hannan RL, Nieves JA, Bolivar J, Dobrolet N, et al. Frequency and indications for tracheostomy and gastrostomy after congenital heart surgery. Pediatr Cardiol. 2009;30:225-31.

10. Chen Q, Langton-Hewer S, Marriage S, Hayes A, Caputo M, Pawade A, et al. Influence of tracheobronchomalacia on outcome of surgery in children with congenital heart disease and its management. Ann Thorac Surg. 2009;88:1970-4.

11. Bertrand P, Navarro H, Caussade S, Holmgren N, Sanchez I. Airway anomalies in children with Down syndrome: endoscopic findings. Pediatr Pulmonol. 2003;36: 137-41.

12. Huang RY, Shapiro NL. Structural airway anomalies in patients with DiGeorge syndrome: a current review. Am J Otolaryngol. 2000;21:326-30.

13. Skinner ML, Halstead LA, Rubinstein CS, Atz AM, Andrews D, Bradley SM. Laryngeal dysfunction after the Norwood procedure. J Thorac Cardiovasc Surg. 2005;130:1293-301.
14. Akay TH, Ozkan S, Gultekin B, Uguz E, Varan B, Sezgin A, et al. Diaphragmatic paralysis after cardiac surgery in children: incidence, prognosis, and surgical management. Pediatr Surg Int. 2006;22:341-6.

15. De Leeuw M, Williams JM, Freedom RM, Williams WG, Shemie SD, McCrindle BW. Impact of diaphragmatic paralysis after cardiothoracic surgery in children. J Thorac Cardiovasc Surg. 1999;118:510-7.

16. Joho-Arreola AL, Bauersfeld U, Stauffer UG, Baenziger O, Bernet V. Incidence and treatment of diaphragmatic paralysis after cardiac surgery in children. Eur J Cardiothorac Surg. 2005;27:53-7.

17. Ovroutski S, Alexi-Meskishvili V, Stiller B, Ewert P, Abdul-Khaliq H, Lemmer J, et al. Paralysis of the phrenic nerve as a risk factor for suboptimal Fontan hemodynamics. Eur J Cardiothorac Surg. 2005;27:561-5.

18. Blot F, Similowski T, Trouillet J, Chardon P, Korach J, Marie-Alyette C, et al. Early tracheotomy versus prolonged endotracheal intubation in unselected severely ill ICU patients. Intensive Care Med. 2008;34:1779-87.

19. Rumbak MJ, Newton M, Truncale T, Schwartz SW, Adams JW, Hazard PB. A prospective, randomized study comparing early percutaneous dilational tracheotomy to prolonged translaryngeal intubation (delayed tracheotomy) in critically ill medical patients. Crit Care Med. 2004;32:1689-94.

20. Bacha EA, Daves S, Hardin J, Abdulla R, Anderson J, Kahana M, et al. Singleventricle palliation for high-risk neonates: the emergence of an alternative hybrid stage I strategy. J Thorac Cardiovasc Surg. 2006;131:163-71.

21. Pizarro C, Murdison KA, Derby CD, Radtke W. Stage II reconstruction after hybrid palliation for high-risk patients with a single ventricle. Ann Thorac Surg. 2008;85:1382-8. 